\title{
A Relation Between a.c. Spectrum of Ergodic Jacobi Matrices and the Spectra of Periodic Approximants *
}

\author{
Y. Last \\ Department of Physics, Technion - Israel Institute of Technology, Haifa, 32000, Israel
}

Received May 27, 1992; in revised form September 10, 1992

\begin{abstract}
We study ergodic Jacobi matrices on $l^{2}(Z)$, and prove a general theorem relating their a.c. spectrum to the spectra of periodic Jacobi matrices, that are obtained by cutting finite pieces from the ergodic potential and then repeating them. We apply this theorem to the almost Mathieu operator: $\left(H_{\alpha, \lambda, \theta} u\right)(n)=u(n+1)+u(n-1)+$ $\lambda \cos (2 \pi \alpha n+\theta) u(n)$, and prove the existence of a.c. spectrum for sufficiently small $\lambda$, all irrational $\alpha$ 's, and a.e. $\theta$. Moreover, for $0 \leq \lambda<2$ and (Lebesgue) a.e. pair $\alpha, \theta$, we prove the explicit equality of measures: $\left|\sigma_{\mathrm{ac}}\right|=|\sigma|=4-2 \lambda$.
\end{abstract}

\section{Introduction}

In this paper, we study one dimensional ergodic Jacobi matrices. These are families of (bounded, self adjoint) operators $H_{\omega}$ on $l^{2}(Z)$, defined by:

$$
\begin{gathered}
H_{\omega}=H_{0}+V_{\omega}, \quad\left(H_{0} u\right)(n)=u(n+1)+u(n-1), \\
\left(V_{\omega} u\right)(n)=V_{\omega}(n) u(n),
\end{gathered}
$$

where $V_{\omega}$ is a (real) stationary bounded ergodic potential, that is: we consider a probability measure space $(\Omega, d p)$, a measure preserving invertible ergodic transformation $T$, and a bounded measurable real-valued function $f$, and define: $V_{\omega}(n)=f\left(T^{n} \omega\right)$.

For such a family $\left\{H_{\omega}\right\}_{\omega \in \Omega}$, it is known [9] that the spectrum of $H_{\omega}$, and its decomposition into a.c., s.c., and p.p. parts are a.e. constant with respect to $\omega$. Namely, there are subsets: $\sigma, \sigma_{\mathrm{ac}}, \sigma_{\mathrm{sc}}, \sigma_{\mathrm{pp}}$ of $R$, such that for a.e. $\omega: \sigma_{\omega} \equiv \operatorname{Spec}\left(H_{\omega}\right)=\sigma$ and $\sigma_{\mathrm{ac}}, \sigma_{\mathrm{sc}}, \sigma_{\mathrm{pp}}$ are (respectively) the absolutely continuous, singular continuous and pure point spectra of $H_{\omega}$ ( $\sigma_{\mathrm{pp}}$ being the closure of the set of eigenvalues).

The Lyapunov exponent $\gamma(E)$, characterizing solutions of the equation:

$$
u(n+1)+u(n-1)+V_{\omega}(n) u(n)=E u(n)
$$

is defined for the family $\left\{H_{\omega}\right\}_{\omega \in \Omega}$, as follows:

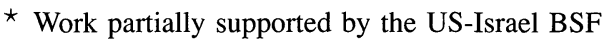


For each $n>0$ define:

$$
\begin{gathered}
T_{n}^{\omega}(E)=\left(\begin{array}{cc}
E-V_{\omega}(n) & -1 \\
1 & 0
\end{array}\right), \\
\Phi_{n}^{\omega}(E)=T_{n}^{\omega}(E) T_{n-1}^{\omega}(E) \ldots T_{1}^{\omega}(E),
\end{gathered}
$$

such that: $\mathbf{u}(n)=\Phi_{n}^{\omega}(E) \mathbf{u}(0)$, where:

$$
\mathbf{u}(n) \equiv\left(\begin{array}{c}
u(n+1) \\
u(n)
\end{array}\right)
$$

and denote:

$$
\gamma_{\omega}(E) \equiv \lim _{n \rightarrow \infty} \frac{1}{n} \ln \left\|\Phi_{n}^{\omega}(E)\right\|
$$

( $\|\cdot\|$ being the operator norm of the $2 \times 2$ matrices). It is a result of Furstenberg and Kesten $[9,13]$, that for every $E$, for a.e. $\omega$ : the limit $\gamma_{\omega}(E)$ exists, and is independent of $\omega$. This (a.e. $\omega$ ) common limit is called the Lyapunov exponent and denoted by $\gamma(E)$.

For the family $\left\{H_{\omega}\right\}_{\omega \in \Omega}$, we define a subset $A$ of $R$ by:

$$
A \equiv\{E \mid \gamma(E)=0\} \text {. }
$$

$A$ is related to $\sigma_{\mathrm{ac}}$ by the Ishii-Pastur-Kotani Theorem [9], which states: $\sigma_{\mathrm{ac}}=\bar{A}^{\text {ess }}$, where: $\bar{A}^{\text {ess }} \equiv\{E \in R|| A \cap(E-\varepsilon, E+\varepsilon) \mid>0 \forall \varepsilon>0\}$, and $|\cdot|$ denotes Lebesgue measure. In particular this theorem implies that $A$ is contained in $\sigma_{\text {ac }}$ up to a set of zero measure, and thus: $\left|\sigma_{\mathrm{ac}}\right| \geq|A|$.

For each $\omega$, we define a sequence $\left\{V_{m}^{\omega}\right\}_{m=1}^{\infty}$ of periodic potentials, by:

$$
\begin{aligned}
V_{m}^{\omega}(n) & =V_{\omega}(n), \quad n=1,2, \ldots, m, \\
V_{m}^{\omega}(n+m) & =V_{m}^{\omega}(n),
\end{aligned}
$$

such that $V_{m}^{\omega}$ is obtained from $V_{\omega}$ by "cutting" a finite piece of length $m$, and then repeating it. For each pair $\omega, m$ we denote by $\sigma_{m}^{\omega}$ the spectrum of the periodic Jacobi matrix: $H_{m}^{\omega} \equiv H_{0}+V_{m}^{\omega}$. As is known for periodic Jacobi matrices, this spectrum consists of $m$ bands (closed intervals). We call $\left\{V_{m}^{\omega}\right\}$ (respectively $\left\{H_{m}^{\omega}\right\}$ ) canonical periodic approximants of $V_{\omega}$ (respectively $H_{\omega}$ ).

The main result of this paper, is the following theorem, which relates the set $A$ (and thus $\sigma_{\mathrm{ac}}$ ) to the spectra of the periodic approximants:

Theorem 1. For a.e. $\omega$ :

$$
\left|\limsup _{m \rightarrow \infty} \sigma_{m}^{\omega} \backslash A\right|=0
$$

where:

$$
\limsup _{m \rightarrow \infty} \sigma_{m}^{\omega} \equiv \bigcap_{l=1}^{\infty} \bigcup_{m=l}^{\infty} \sigma_{m}^{\omega}
$$

Since $\left|\bigcup_{m=1}^{\infty} \sigma_{m}^{\omega}\right|<\infty$, we have (see e.g. [21]): $\left|\lim \sup \sigma_{m}^{\omega}\right| \geq \lim \sup \left|\sigma_{m}^{\omega}\right|$, and thus Theorem 1 implies:

Corollary 1.1. For a.e. $\omega$ :

$$
\limsup _{m \rightarrow \infty}\left|\sigma_{m}^{\omega}\right| \leq|A| \leq\left|\sigma_{\mathrm{ac}}\right| .
$$


If the potential is "highly random," it is known [9], that there is no absolutely continuous spectrum, and thus Corollary (1.1) simply implies that for such potentials: $\lim \sup \left|\sigma_{m}^{\omega}\right|=0$ for a.e. $\omega$. For certain almost periodic potentials, however, a.c. spectrum does occur, and Theorem 1 (or Corollary 1.1) can serve as a tool for establishing its existence and estimating its Lebesgue measure.

In this paper we will apply Theorem 1 to the study of the almost Mathieu operator $H_{\alpha, \lambda, \theta}$, defined by:

$$
H_{\alpha, \lambda, \theta}=H_{0}+V_{\alpha, \lambda, \theta}, \quad V_{\alpha, \lambda, \theta}(n)=\lambda \cos (2 \pi \alpha n+\theta) .
$$

It is known [9] that if $\alpha$ is irrational, then the family: $\left\{H_{\alpha, \lambda, \theta}\right\}_{\theta \in[0,2 \pi]}$ is ergodic in the sense discussed above, if we take Lebesgue measure for the measure $d p$ on $\Omega=[0,2 \pi]$ (i.e. $d p(\theta)=d \theta$ ). The spectrum of $H_{\alpha, \lambda, \theta}$, denoted by $\sigma(\alpha, \lambda)$, is known in this case ( $\alpha$ irrational) to be completely independent of $\theta$, and the sets $\sigma_{\mathrm{ac}}(\alpha, \lambda), \sigma_{\mathrm{sc}}(\alpha, \lambda)$, and $\sigma_{\mathrm{pp}}(\alpha, \lambda)$ are defined for the family $\left\{H_{\alpha, \lambda, \theta}\right\}_{\theta \in[0,2 \pi]}$, being the (Lebesgue) a.e. $\theta$ independent a.c., s.c., and p.p. spectra of $H_{\alpha, \lambda, \theta}$. The set $A(\alpha, \lambda)$ is also defined for the family, as the set where the Lyapunov exponent vanishes.

The almost Mathieu operator was extensively studied by many authors [1-5, 7, $8,11,12,18,19]$, and many of its spectral characteristics are known. Bellissard, Lima, and Testard [4] have shown that for (Lebesgue) a.e. $\alpha$, all $\theta$ and sufficiently small coupling $\lambda, H_{\alpha, \lambda, \theta}$ has some a.c. spectrum, and moreover for a.e. irrational $\alpha:\left|\sigma_{\mathrm{ac}}(\alpha, \lambda)\right| \rightarrow 4$ as $\lambda \rightarrow 0$. Chulaevsky and Delyon [8] have recently shown that for a.e. pair $\alpha, \theta$ and sufficiently small $\lambda, H_{\alpha, \lambda, \theta}$ has purely a.c. spectrum. In this paper we combine Theorem 1 with some results of Avron, van Mouche, and Simon [3] to show:

Theorem 2. (i) For all irrational $\alpha$ 's:

$$
\left|\sigma_{\mathrm{ac}}(\alpha, \lambda)\right| \geq|A(\alpha, \lambda)| \geq 4-\left(2+\frac{\pi}{\sqrt{5}}\right) \lambda .
$$

(ii) If $0 \leq \lambda<2$ and $\alpha$ is an irrational obeying.

$$
\lim _{n \rightarrow \infty} q_{n}^{2}\left|\alpha-\frac{p_{n}}{q_{n}}\right|=0
$$

for some sequence $\left\{p_{n} / q_{n}\right\}_{n=1}^{\infty}$ of rationals, then:

$$
|A(\alpha, \lambda)|=\left|\sigma_{\mathrm{ac}}(\alpha, \lambda)\right|=|\sigma(\alpha, \lambda)|=4-2 \lambda .
$$

Remarks. 1) The set of irrationals characterized in (ii), has full Lebesgue measure [14].

2) The equality $|\sigma(\alpha, \lambda)|=4-2 \lambda$ was conjectured by Aubry and Andre [1] to hold for every irrational $\alpha$ and $0 \leq \lambda \leq 2$, and was studied by Thouless [19] and by Avron, van Mouche, and Simon [3]. While they did not succeed to prove it for the general case, and only obtained the lower bound: $|\sigma(\alpha, \lambda)| \geq 4-2 \lambda$, we will show that for $\lambda \neq 2$, and a.e. $\alpha$ (i.e. $\alpha$ as in (ii)), it does follow immediately from the results of Avron et al.

Theorem 2 extends the previously known results, giving explicit measures for "most" $\alpha$ 's, and establishing the existence of a.c. spectrum (at sufficiently small coupling) for all $\alpha$ 's. The somewhat striking part of this extension, is the existence of a.c. spectrum for the case where $\alpha$ is a Liouville number. In this case the spectrum was previously conjectured to be purely singular continuous $[10,17]$, and the existence of 
the a.c. spectrum disproves a general conjecture of Deift and Simon about the existence of certain types of eigenfunctions associated to a.c. spectra of ergodic Jacobi matrices (see Sect. 7 of [10] for details).

In Sect. 2 we describe some preliminaries from the theory of periodic Jacobi matrices. In Sect. 3 we prove Theorem 1, and in Sect. 4 we prove Theorem 2.

It is a pleasure to thank Prof. J. Avron for much support and useful discussions.

\section{Preliminaries}

In this section, we describe some basic results from the theory of periodic Jacobi matrices. The description is brief, and mostly without proofs. For further details the reader is referred to [20] and [16].

Let $V=\{V(n)\}$ be a periodic potential with period $p$ (i.e. $V(n+p)=V(n)$ for all $n$ ). We denote: $H \equiv H_{0}+V, \sigma \equiv \operatorname{Spec}(H)$, and define the $2 \times 2$ transfer matrices $\Phi_{n}(E)$, by (1.3), with $V_{\omega}=V$. From the periodicity of $V$, we have:

$$
\lim _{n \rightarrow \infty}\left\|\Phi_{n}(E)\right\|^{1 / n}=\left[\operatorname{Spr}\left(\Phi_{p}(E)\right)\right]^{1 / p},
$$

where $\operatorname{Spr}(\cdot)$ is the spectral radius. Thus, the Lyapunov exponent $\gamma(E)$ exists (for the individual operator $H$ ) for every $E \in R$, and is determined by the one period transfer matrix $\Phi_{p}(E)$ as:

$$
\gamma(E) \equiv \lim _{n \rightarrow \infty} \frac{1}{n} \ln \left\|\Phi_{n}(E)\right\|=\frac{1}{p} \ln \left[\operatorname{Spr}\left(\Phi_{p}(E)\right)\right] .
$$

Since $\operatorname{det} \Phi_{n}(E)=1$ (for all $n$ ), we see that for $E \in A \equiv\{E \mid \gamma(E)=0\} \Phi_{p}(E)$ has eigenvalues presentable as: $e^{ \pm i k p}$ (with $k \in R$ ), and for $E \notin A$ it has eigenvalues presentable as: $e^{ \pm \gamma p}$. The corresponding eigenvectors give rise (as initial conditions) to solutions $\left\{u^{ \pm}(n)\right\}_{n=-\infty}^{\infty}$ of Eq. (1.2) (with $V_{\omega}=V$ ), obeying: $u^{ \pm}(n+p)=$ $e^{ \pm i k p} u^{ \pm}(n)$ for $E \in A$, and $u^{ \pm}(n+p)=e^{ \pm \gamma p} u^{ \pm}(n)$ for $E \notin A$. From this, it is not hard to verify the existence of $(E-H)^{-1}$ (as a bounded operator) for $E \notin A$, and its inexistence for $E \in A$. Thus, we have: $\sigma=A$.

The solutions obeying: $u^{ \pm}(n+p)=e^{ \pm i k p} u^{ \pm}(n)$, that characterize $\sigma$, are called Bloch wave solutions, and the corresponding (real) $k$ 's are called Bloch wave numbers. The existence of these solutions for every $E \in \sigma$, allows to transform the spectral problem for $H$ into a finite matrix eigenvalue problem, by defining:

$$
\mathscr{B}(k) \equiv\left(\begin{array}{ccccc}
V(1) & 1 & & & e^{-i k p} \\
1 & V(2) & 1 & & \\
& 1 & \ddots & \ddots & \\
& & \ddots & \ddots & 1 \\
e^{i k p} & & & 1 & V(p)
\end{array}\right)
$$

and noting that:

$$
\sigma=\bigcup_{k} \operatorname{Spec}(\mathscr{A}(k))
$$

By direct expansion in minors, it can be shown that:

$$
\operatorname{det}(E-\mathscr{f}(k))=\Delta(E)-2 \cos k p,
$$


where $\Delta(E)$ (called the discriminant, and easily shown to coincide with the trace of $\left.\Phi_{p}(E)\right)$ is a polynomial of degree $p$, independent of $k$, with real coefficients and $p$ distinct real zeroes. Moreover, (for $E \in R$ ) we have: $|\Delta(E)| \geq 2$ in all the extremum points of $\Delta(E)$. This shows that $\sigma$ consists of $p$ bands (closed intervals), such that $\Delta(E)$ is strongly monotone on each band. By considering only $k \in[0, \pi / p]$ (which is always assumed throughout the rest of this paper), (2.5) defines $p$ dispersion functions: $E_{l}(k), l=1,2, \ldots, p$, such that each $E_{l}(k)$ is a strongly monotone $C^{\infty}$ function of $k$, from the interval $[0, \pi / p]$ onto the $l^{\text {th }}$ band of $\sigma$. In particular the width of the $l^{\text {th }}$ band is given by: $\int_{0}^{\pi / p}\left|\frac{d E_{l}(k)}{d k}\right| d k$.

We denote by $\operatorname{Intb}(\sigma)$ the union of the interiors of the bands of $\sigma$. For each $E \in \operatorname{Intb}(\sigma)$ there is a unique pair $l, k$, such that $E=E_{l}(k)$, and moreover there is a unique corresponding derivative: $\frac{d E_{l}(k)}{d k}$, which we denote simply by: $\frac{d E}{d k}$. We have (Lemma 4.1 of [16]):

Proposition 2.1. Let $E \in \operatorname{Intb}(\sigma)$, and let $\{u(n)\}$ be a corresponding Bloch wave solution of Eq. (1.1). normalized such that:

$$
\sum_{n=1}^{p}|u(n)|^{2}=1
$$

then for each $n$ :

$$
\left|\frac{d E}{d k}\right|=2 p|\operatorname{Im}[u(n) \overline{u(n+1)}]|,
$$

where - denotes complex conjugation.

\section{Proof of Theorem 1}

Lemma 3.1. For every $\omega$ and $m \geq 2$, let $E \in \operatorname{Intb}\left(\sigma_{m}^{\omega}\right)$, and let $\Phi_{m}^{\omega}(E)$ be the $2 \times 2$ transfer matrix defined by (1.3), then:

$$
\left\|\Phi_{m}^{\omega}(E)\right\| \leq 2 m\left|\frac{d E}{d k_{m}^{\omega}}\right|^{-1}
$$

where $k_{m}^{\omega}$ is the appropriate Bloch wave number.

Proof. Since $E \in \operatorname{Intb}\left(\sigma_{m}^{\omega}\right)$, and since $\Phi_{m}^{\omega}(E)$ is a one period transfer matrix for $H_{m}^{\omega}$, it has two eigenvalues: $e^{ \pm i k_{m}^{\omega} m}$, and corresponding normalized eigenvectors: $\mathbf{x}^{ \pm}=\left(x_{1}^{ \pm}, x_{2}^{ \pm}\right)^{T}$, which are uniquely defined up to a phase. The eigenvectors $\mathbf{x}^{ \pm}$ can be chosen to be complex conjugates of each other, and thus we can assume: $x_{1}^{+}=x_{1}, x_{2}^{+}=x_{2}, x_{1}^{-}=\overline{x_{1}}, x_{2}^{-}=\overline{x_{2}}$, where $\left|x_{1}\right|^{2}+\left|x_{2}\right|^{2}=1$. Let $a, b \in C$ obey: $|a|^{2}+|b|^{2}=1$, and denote: $\mathbf{y}=a \mathbf{x}^{+}+b \mathbf{x}^{-}$, then we have: 


$$
\begin{aligned}
\frac{\left\|\Phi_{m}^{\omega}(E) \mathbf{y}\right\|^{2}}{\|\mathbf{y}\|^{2}} & =\frac{\left\|a e^{i k_{m}^{\omega} m} \mathbf{x}^{+}+b e^{-i k_{m}^{\omega} m} \mathbf{x}^{-}\right\|^{2}}{\left\|a \mathbf{x}^{+}+b \mathbf{x}^{-}\right\|^{2}} \\
& \leq \frac{(|a|+|b|)^{2}}{|a|^{2}+|b|^{2}-2|a||b|\left|\left\langle\mathbf{x}^{+}, \mathbf{x}^{-}\right\rangle\right|} \\
& \leq \frac{2}{1-\left|\left\langle\mathbf{x}^{+}, \mathbf{x}^{-}\right\rangle\right|} \\
& =\frac{2\left(1+\left|\left\langle\mathbf{x}^{+}, \mathbf{x}^{-}\right\rangle\right|\right)}{1-\left|\left\langle\mathbf{x}^{+}, \mathbf{x}^{-}\right\rangle\right|^{2}} \\
& \leq \frac{4}{1-\left|\left\langle\mathbf{x}^{+}, \mathbf{x}^{-}\right\rangle\right|^{2}} .
\end{aligned}
$$

Denote by $\varphi$ the phase difference between $x_{1}$ and $x_{2}$, such that: $x_{1} \overline{x_{2}}=\left|x_{1}\right|\left|x_{2}\right| e^{i \varphi}$, then:

$$
\begin{aligned}
1-\left|\left\langle\mathbf{x}^{+}, \mathbf{x}^{-}\right\rangle\right|^{2} & =\left(\left|x_{1}\right|^{2}+\left|x_{2}\right|^{2}\right)^{2}-\left|x_{1}^{2}+x_{2}^{2}\right|^{2} \\
& =2\left|x_{1}\right|^{2}\left|x_{2}\right|^{2}-2 \operatorname{Re}\left(x_{1}^{2} \overline{x_{2}^{2}}\right) \\
& =2\left|x_{1}\right|^{2}\left|x_{2}\right|^{2}(1-\cos 2 \varphi) \\
& =4\left|x_{1}\right|^{2}\left|x_{2}\right|^{2} \sin ^{2} \varphi \\
& =4\left(\operatorname{Im}\left(x_{1} \overline{x_{2}}\right)\right)^{2}
\end{aligned}
$$

and thus (3.1) implies:

$$
\left\|\Phi_{m}^{\omega}(E)\right\| \leq \frac{1}{\left|\operatorname{Im}\left(x_{1} \overline{x_{2}}\right)\right|}
$$

As described in Sect. 2, we have: $x_{1}=u(1), x_{2}=u(0)$, for some Bloch wave solution $\{u(n)\}$ of Eq. (1.1) (with $V_{\omega}=V_{m}^{\omega}$ ). Since $\left|x_{1}\right|^{2}+\left|x_{2}\right|^{2}=1$, we have:

$$
N^{2} \equiv \sum_{n=1}^{m}|u(n)|^{2} \geq 1
$$

and $\{u(n) / N\}$ is a normalized (over one period) Bloch wave solution. Thus, Proposition 2.1 implies:

$$
\left|\frac{d E}{d k_{m}^{\omega}}\right|=\frac{2 m}{N^{2}}\left|\operatorname{Im}\left(x_{1} \overline{x_{2}}\right)\right|
$$

and from (3.3), we obtain:

$$
\left\|\Phi_{m}^{\omega}(E)\right\| \leq \frac{2 m}{N^{2}}\left|\frac{d E}{d k_{m}^{\omega}}\right|^{-1} \leq 2 m\left|\frac{d E}{d k_{m}^{\omega}}\right|^{-1} .
$$

Proof of Theorem 1. For every $\omega, m \geq 2$ and $\varepsilon>0$, we define:

$$
S_{m}^{\omega}(\varepsilon) \equiv\left\{E\left|E \in \operatorname{Intb}\left(\sigma_{m}^{\omega}\right),\right| \frac{d E}{d k_{m}^{\omega}} \mid \geq \varepsilon\right\},
$$

and for each $\omega$, we define:

$$
S_{\omega} \equiv \limsup _{m \rightarrow \infty} S_{m}^{\omega}\left(m^{-2}\right)
$$


Since there are $m$ bands in $\sigma_{m}^{\omega}$, and $0 \leq k_{m}^{\omega} \leq \pi / m$ for each band, we clearly have:

$$
\left|\sigma_{m}^{\omega} \backslash S_{m}^{\omega}(\varepsilon)\right|<\pi \varepsilon
$$

from which follows that the sum $\sum_{m=2}^{\infty}\left|\sigma_{m}^{\omega} \backslash S_{m}^{\omega}\left(m^{-2}\right)\right|$ converges. As can be easily seen from the definition of lim sup, this implies:

$$
\left|\limsup _{m \rightarrow \infty}\left(\sigma_{m}^{\omega} \backslash S_{m}^{\omega}\left(m^{-2}\right)\right)\right|=0 .
$$

Thus, since: $\lim \sup \sigma_{m}^{\omega} \backslash S_{\omega} \subseteq \lim \sup \left(\sigma_{m}^{\omega} \backslash S_{m}^{\omega}\left(m^{-2}\right)\right)$, and clearly: $S_{\omega} \subseteq \lim \sup \sigma_{m}^{\omega}$, we have:

$$
\limsup _{m \rightarrow \infty} \sigma_{m}^{\omega} \approx S_{\omega}
$$

where $\approx$ denotes set equality up to sets of zero (Lebesgue) measure.

Let $E \in S_{\omega}$, then $E \in S_{m_{l}}^{\omega}\left(m_{l}^{-2}\right)$ for some subsequence $\left\{m_{l}\right\}_{l=1}^{\infty}$ of positive integers. From (3.7) and Lemma 3.1 we have for all $l$ :

$$
\left\|\Phi_{m_{l}}^{\omega}(E)\right\| \leq 2 m_{l}^{3}
$$

which implies:

$$
\liminf _{m \rightarrow \infty} \frac{1}{m} \ln \left\|\Phi_{m}^{\omega}(E)\right\|=0 .
$$

From the Furstenberg-Kesten Theorem [9, 13], we know that for a.e. $\omega$, for a.e. $E$, the limit: $\lim \frac{1}{m} \ln \left\|\Phi_{m}^{\omega}(E)\right\|$ exists, and is equal to $\gamma(E)$. Therefore, we obtain for a.e. $\omega$, for a.e. $E \in S_{\omega}: \gamma(E)=0$. From (3.11), this implies that for a.e. $\omega$, for a.e. $E \in \lim \sup \sigma_{m}^{\omega}: \gamma(E)=0$, and this is precisely the statement of Theorem 1 .

\section{Proof of Theorem 2}

If $\alpha=p / q$ is a rational number, then for each $\theta$, the almost Mathieu operator defined by (1.8), is a periodic Jacobi matrix with period $q$, and $\theta$ dependent spectrum: $\sigma(p / q, \lambda, \theta)$. For every irrational $\alpha, \theta \in[0,2 \pi]$ and $\lambda \geq 0$, denote by: $\left\{\sigma_{m}(\alpha, \lambda, \theta)\right\}_{m=1}^{\infty}$, the spectra of the canonical periodic approximants of $H_{\alpha, \lambda, \theta}$, as defined in Sect. 1, then:

Lemma 4.1. For every sequence of rationals: $\alpha_{j}=p_{j} / q_{j} \rightarrow \alpha$, there is a sequence $\left\{\theta_{j}\right\}_{j=1}^{\infty}$, such that:

$$
|| \sigma_{q_{j}}(\alpha, \lambda, \theta)|-| \sigma\left(\alpha_{j}, \lambda, \theta_{j}|| \leq \pi \lambda q_{j}^{2}\left|\alpha-\alpha_{j}\right| .\right.
$$

Proof. Both $H_{\alpha_{j}, \lambda, \theta_{j}}$ and the $q_{j}^{\text {th }}$ canonical periodic approximant of $H_{\alpha, \lambda, \theta}$, are periodic Jacobi matrices with period $q_{j}$, and the difference of the measures of their spectra must be smaller or equal to the sum of the distances between their corresponding band edges. These band edges are eigenvalues of the corresponding $\mathscr{A}(k)$ matrices with $k=0$ and $k=\pi / q$, (see Sect. 2). Since the difference of two corresponding (i.e. with the same $k) \mathscr{A}(k)$ matrices is diagonal, it follows from Lidskii's Theorem $[6,15]$, that the sum of the distances between their corresponding 
eigenvalues must be smaller or equal to the sum of the absolute values of the (diagonal) entries of the difference matrix. This implies:

$$
\begin{aligned}
|| \sigma_{q_{j}}(\alpha, \lambda, \theta)|-| \sigma\left(\alpha_{j}, \lambda, \theta_{j}\right)|| & \leq 2 \sum_{n=1}^{q_{j}}\left|\lambda \cos (2 \pi \alpha n+\theta)-\lambda \cos \left(2 \pi \alpha_{\jmath} n+\theta_{j}\right)\right| \\
& \leq 2 \lambda \sum_{n=1}^{q_{j}}\left|2 \pi\left(\alpha-\alpha_{j}\right) n+\left(\theta-\theta_{j}\right)\right|
\end{aligned}
$$

By choosing $\theta_{j}$ such that: $\left(\theta-\theta_{j}\right)=-\pi\left(\alpha-\alpha_{j}\right)\left(q_{j}+1\right)$, the last sum (which is the sum of two arithmetic series) is seen to be smaller than $\pi \lambda\left|\alpha-\alpha_{j}\right| q_{j}^{2}$, and this proves the lemma.

For every real $\alpha, \lambda$, we denote:

$$
S(\alpha, \lambda) \equiv \bigcup_{\theta} \operatorname{Spec}\left(H_{\alpha, \lambda, \theta}\right) .
$$

If $\alpha$ is irrational then we have: $S(\alpha, \lambda)=\sigma(\alpha, \lambda)$, and if $\alpha=p / q$ is rational then the set $S(\alpha, \lambda)$ is similar to $\sigma(\alpha, \lambda, \theta)$, consisting of $q$ bands. We denote by $G(\alpha, \lambda)$ the union of the gaps in $S(\alpha, \lambda)$, such that:

$$
|S(\alpha, \lambda)|=\max S(\alpha, \lambda)-\min S(\alpha, \lambda)-|G(\alpha, \lambda)| \text {. }
$$

Avron, van Mouche, and Simon [3], proved the following:

Proposition 4.1. (i) For every rational $p / q, \lambda \geq 0$ and $\theta \in[0,2 \pi]$ :

$$
|\sigma(p / q, \lambda, \theta)| \geq 4-2 \lambda \text {. }
$$

(ii) For every $0 \leq \lambda<2$, and a sequence of rationals $\alpha_{j}=p_{j} / q_{\jmath}$, obeying: $q_{j} \rightarrow \infty$ with $p_{j}, q_{j}$ relatively prime:

$$
\lim _{j \rightarrow \infty}\left|S\left(\alpha_{j}, \lambda\right)\right|=4-2 \lambda .
$$

(iii) For every $\lambda>0$, there is a constant $C$, such that if $\left|\alpha-\alpha^{\prime}\right|<C$, then for every gap in $S(\alpha, \lambda)$ with midpoint $E_{g}$, and measure $|g|$ larger than $12\left(\lambda\left|\alpha-\alpha^{\prime}\right|\right)^{1 / 2}$, there is a corresponding (containing $E_{g}$ ) gap in $S\left(\alpha^{\prime}, \lambda\right)$ with measure larger than:

$$
|g|-12\left(\lambda\left|\alpha-\alpha^{\prime}\right|\right)^{1 / 2} \text {. }
$$

(iv) The same continuity as in (iii) also holds for the extreme edges of $S(\alpha, \lambda)$, namely for $\left|\alpha-\alpha^{\prime}\right|<C$ :

$$
\left|\min _{\min } S(\alpha, \lambda)-\operatorname{mix}_{\min } S\left(\alpha^{\prime}, \lambda\right)\right|<6\left(\lambda\left|\alpha-\alpha^{\prime}\right|\right)^{1 / 2} .
$$

Proof of Theorem 2. (i) Let $\alpha$ be any irrational, then it is known [14] that there is a sequence of rationals: $\alpha_{j}=p_{j} / q_{j} \rightarrow \alpha$, obeying: $q_{j}^{2}\left|\alpha-\alpha_{j}\right| \leq 1 / \sqrt{5}$. Thus, from statement (i) of Proposition 4.1 and from Lemma 4.1 we obtain:

$$
\begin{aligned}
\left|\sigma_{q_{j}}(\alpha, \lambda, \theta)\right| & \geq 4-2 \lambda-\pi \lambda q_{j}^{2}\left|\alpha-\alpha_{j}\right| \\
& \geq 4-2 \lambda-\frac{\pi \lambda}{\sqrt{5}} \\
& =4-\left(2+\frac{\pi}{\sqrt{5}}\right) \lambda .
\end{aligned}
$$


Since $q_{j} \rightarrow \infty$ as $j \rightarrow \infty$, this implies (for every $\theta$ ):

$$
\limsup _{m \rightarrow \infty}\left|\sigma_{m}(\alpha, \lambda, \theta)\right| \geq 4-\left(2+\frac{\pi}{\sqrt{5}}\right) \lambda,
$$

and from Corollary 1.1 the statement follows.

(ii) By repeating the arguments of (i), but with the sequence of rationals obeying: $q_{j}^{2}\left|\alpha-\alpha_{j}\right| \rightarrow 0$, we clearly obtain: $|A(\alpha, \lambda)| \geq 4-2 \lambda$. In order to complete the proof it is sufficient to show: $|\sigma(\alpha, \lambda)| \leq 4-2 \lambda$. Since there are at most $q_{j}-1$ gaps in $S\left(\alpha_{j}, \lambda\right)$, we obtain from statement (iii) of Proposition 4.1 (for $\left|\alpha-\alpha_{j}\right|<C$ ):

$$
|G(\alpha, \lambda)|>\left|G\left(\alpha_{j}, \lambda\right)\right|-12\left(q_{j}-1\right)\left(\lambda\left|\alpha-\alpha_{j}\right|\right)^{1 / 2},
$$

and from (4.3) and statement (iv) of Proposition 4.1, this implies:

$$
|S(\alpha, \lambda)|<\left|S\left(\alpha_{j}, \lambda\right)\right|+12 q_{j}\left(\lambda\left|\alpha-\alpha_{\jmath}\right|\right)^{1 / 2} .
$$

As $j \rightarrow \infty$ we have from statement (ii) of Proposition 4.1: $\left|S\left(\alpha_{j}, \lambda\right)\right| \rightarrow 4-2 \lambda$, and by our assumption on $\left\{\alpha_{j}\right\}: q_{j}\left|\alpha-\alpha_{j}\right|^{1 / 2} \rightarrow 0$. Thus, (4.7) implies:

$$
|\sigma(\alpha, \lambda)|=|S(\alpha, \lambda)| \leq 4-2 \lambda,
$$

which completes the proof.

\section{References}

1. Aubry, S., Andre, G.: Analyticity breaking and Anderson localization in incommensurate lattices. Ann. Israel Phys. Soc. 3, 133-164 (1980)

2. Avron, J., Simon, B.: Almost periodic Schrödinger operators. II. The integrated density of states. Duke Math. J. 50, 369-391 (1983)

3. Avron, J., van Mouche, P., Simon, B.: On the measure of the spectrum for the almost Mathieu operator. Commun. Math. Phys. 132, 103-118 (1990)

4. Bellissard, J., Lima, R., Testard, D.: A metal-insulator transition for the almost Mathieu model. Commun. Math. Phys. 88, 207-234 (1983)

5. Bellissard, J., Simon, B.: Cantor spectrum for the almost Mathieu equation. J. Funct. Anal. 48, 408-419 (1982)

6. Bhatia, R.: Perturbation bounds for matrix eigenvalues. Pitman Research Notes in Mathematics Series 162, Essex: Longman 1987

7. Choi, M.D., Elliott, G.A., Yui, N.: Gauss polynomials and the rotation algebra. Invent. Math. 99, 225-246 (19909)

8. Chulaevsky, V., Delyon, F.: Purely absolutely continuous spectrum for almost Mathieu operators. J. Stat. Phys. 55, 1279-1284 (1989)

9. Cycon, H.L., Froese, R.G., Kirsch, W., Simon, B.: Schrödinger operators. Berlin, Heidelberg, New York: Springer 1987

10. Deift, P., Simon, B.: Almost periodic Schrödinger operators. III. The absolutely continuous spectrum in one dimension. Commun. Math. Phys. 90, 389-411 (1983)

11. Delyon, F.: Absence of localization for the almost Mathieu equation. J. Phys. A 20, L 21-L 23 (1987)

12. Fröhlich, J., Spencer, T., Wittwer, P.: Localization for a class of one dimensional quasi-periodic Schrödinger operators. Commun. Math. Phys. 132, 5-25 (1990)

13. Furstenberg, H., Kesten, H.: Products of random matrices. Ann. Math. Stat. 31, 457-469 (1960)

14. Hardy, G.H., Wright, E.M.: An introduction to the theory of numbers, Fifth ed. Oxford: Oxford University Press 1979

15. Kato, T.: Perturbation theory for linear operators. Berlin, Heidelberg, New York: Springer 1966 
16. Last, Y.: On the measure of gaps and spectra for discrete 1D Schrödinger operators. Commun. Math. Phys. (to appear)

17. Simon, B.: Almost periodic Schrödinger operators. A review. Adv. Appl. Math. 3, 463-490 (1982)

18. Sinai, Ya.G.: Anderson localization for one-dimensional difference Schrödinger operator with quasiperiodic potential. J. Stat. Phys. 46, 861-909 (1987)

19. Thouless, D.J.: Bandwidth for a quasiperiodic tight binding model. Phys. Rev. B 28, 4272-4276 (1983)

20. Toda, M.: Theory of nonlinear lattices. 2nd. ed., chap. 4. Berlin, Heidelberg, New York: Springer 1989

21. Zaanen, A.C.: An introduction to the theory of integration. Amsterdam: North-Holland 1958

Communicated by B. Simon 